\title{
Evoluzione nel trattamento delle fratture diafisarie del bambino
}

R. Facchini

Clinica Ortopedica dell'Università-C.T.0., Milano

ABSTRACT Evolution in the management of pediatric shaft fractures

Social, economic and technological changes produced a significant increase of surgical management of paediatric shaft fractures. The Author analyzes how guidelines changed during the latest 30 years.

In occasione del Congresso EPOS di Zagabria (2010) sono stato par ticolarmente interessato al lavoro "Operative treatment of fractures in children is increasing" del gruppo finlandese di Helenius e coll. [1].

Mentre condividevo pienamente le argomentazioni della loro presentazione, ripercorrevo mentalmente le tappe più significative della prima parte della mia carriera. In effetti, negli anni Settanta il nostro sforzo era indirizzato a differenziare il più possibile la traumatologia del bambino da quella dell'adulto, individuando tutte le caratteristiche anatomiche, fisiologiche e biologiche del lo scheletro del soggetto in accrescimento. I risultati dei nostri studi e delle nostre ricerche venivano nella pratica trasferiti nei protocolli di trattamento delle fratture [2]. Queste venivano classificate in rapporto alla sede anatomica, al tipo di rima, alla posizione del focolaio, all'età del paziente e alle sue potenzialità di crescita residua.

Il nostro sforzo, rivolto in particolare alle fratture diafisarie delle ossa lunghe, era finalizzato alla scelta del trattamento meno invasivo possibile. Pertanto venivano considerate tutte le possibili complicazioni derivanti dal trattamento cruento [3], individuando, per esempio, la specifica complicazione dell'iperallungamento direttamente proporzionale al l'invasività del trattamento eseguito.

Al contrario, veniva evidenziata una serie di fattori a sostegno della maggiore affidabilità del trattamento conservativo. In effetti le fratture del bambino presentano tempi rapidi di consolidazione [4], non risentono negativamente dell'immobilizzazione in gesso, non richiedono fisioterapia a recupero della funzione e, sfruttando la crescita residua, consentono rimodellamenti a distanza tali da non dover ricercare necessariamente riduzioni anatomiche della frattura stessa [5]

Tutto ciò indirizzava l'ortopedico pediatrico al trattamento conservativo, sapendo però che tutte le situazioni favorevoli sopra menzionate erano in diretta funzione dell'età del bambino, del segmento fratturato, della sede della frattura e del tipo di scomposizione.

La ricerca diveniva allora estremamente sofisticata nel differen ziare l'età anagrafica da quella biologica e soprattutto nel cercare di individuare fasce di età omogenee che consentissero maggiore o minore tolleranza nel trattamento delle fratture. Venivano pertanto studiati i tempi medi di consolidazione per tipo di segmento, per fascia di età, per sede di frattura, per tipo di scomposizione iniziale, per grado di scomposizione residua dopo il trattamento, calcolando la possibilità di rimodellamento spontaneo del segmento interessato in funzione dell'età e, più precisamente, in funzione delle potenzialità residue di crescita [6]

È stata quindi l'epoca trionfale de trattamento conservativo, i cui cardini erano rappresentati dalla riduzione incruenta, meglio se estemporanea o, eventualmente, con trazione continua, trans-scheletrica o, nei più piccoli, di superficie, e successiva immobilizzazione in apparecchio gessato. A cavallo fra la fine degli anni Ottanta e i primi anni Novanta si è verificata la svolta. In Italia si è prepotentemente imposta la fissazione esterna propugnata da Nando de Sanctis e sostenuta dalla imponente casistica del Santobono di Napoli. Nello stesso periodo la scuola francese di Nancy diffondeva l'utilizzo del chiodo elastico Successivamente sono state introdotte anche placche $\mathrm{di}$ nuova concezione che, agendo da fissatore interno, minimizzano la stimolazione del periostio, adattandosi pertanto a un uso anche nelle diafisi di soggetti ancora in accrescimento.

Queste nuove metodiche, ac compagnate dai notevoli progressi delle tecniche anestesiologiche e dalla migliorata assistenza perioperatoria, hanno dato il via a un progressivo aumento delle indicazioni al trattamento chirurgico delle fratture diafisarie del bambino Non solo: a queste argomentazioni, squisitamente tecniche, si sono presto affiancate motivazioni di altra natura. Infatti, accanto alle regole auree e sempre valide di limitare il dolore, correggere le deviazioni assiali e rotatorie, consentire una rapida ripresa funzionale, ottenere una guarigione completa con il minimo di complicazioni e restituire infine autonomia al piccolo paziente, si sono poi aggiunte la necessità di ridurre i tempi di ospedalizzazione, di produrre il minor fastidio per il paziente e il minor disagio per la famiglia. E se è condivisibile con la famiglia il cercare di limitare l'uso di un apparecchio gessato pelvi-podalico o toraco-brachiale, certamente meno condivisibile è la pretesa di una soluzione immediata del problema con una chirurgia miracolosamente riparatrice. Non solo, la deriva chirurgica si è così radicata da innescare problemi di medicina difensiva, senza contare che comunemente l'ortopedico viene valutato, purtroppo spesso anche da colleghi o da D.G. di Aziende Ospedaliere, sulla base del numero di interventi chirurgici eseguiti.

Sembrano ormai lontanissimi tempi in cui il Maestro ci insegnava che il miglior ortopedico è quello che opera di meno, in quanto sa risolvere i problemi senza dover ricorrere alla chirurgia. Gli apparecchi gessati, un tempo vanto della tradizione ortopedica italiana, per quanto restino l'immobilizzazione ideale nel bambino - che necessita di tempi di in gessatura più brevi, ben li tollera e poco risente comunque anche di lunghe immobilizzazioni - sembrano aver fatto ormai la loro storia, spesso sostituiti dalla chirurgia e da tutori preformati, apparentemente meno "costosi" e "socialmente" più tollerati.

I media, diffondendo a volte in modo distorto i concetti di mini-

\begin{tabular}{|c|c|c|c|}
\hline Sito di frattura & Età (anni) & Scelta di trattamento & Metodologia di trattamento \\
\hline Femore & $\begin{array}{l}0-2 \\
2-6 \\
6-10 \\
10-12 \\
>12\end{array}$ & $\begin{array}{l}\text { Incruenta } \\
\text { Incruenta o chirurgica } \\
\text { Incruenta o chirurgica } \\
\text { Incruenta o chirurgica } \\
\text { Incruenta o chirurgica }\end{array}$ & $\begin{array}{l}\text { Apparecchio gessato } \pm \text { trazione } \\
\text { Gesso o fissatore esterno } \pm \text { trazione } \\
\text { Fissatore esterno o sintesi } \pm \text { trazione } \\
\text { Fissatore esterno o sintesi } \\
\text { Sintesi interna }\end{array}$ \\
\hline Gamba & $\begin{array}{l}0-2 \\
2-6 \\
6-10 \\
10-12 \\
>12\end{array}$ & $\begin{array}{l}\text { Incruenta o chirurgica } \\
\text { Incruenta o chirurgica } \\
\text { Incruenta o chirurgica } \\
\text { Incruenta o chirurgica } \\
\text { Incruenta o chirurgica }\end{array}$ & $\begin{array}{l}\text { Apparecchio gessato } \pm \text { trazione } \\
\text { Apparecchio gessato } \pm \text { trazione } \\
\text { Gesso o fissatore } \pm \text { trazione } \\
\text { Gesso o fissatore } \\
\text { Sintesi interna } 0 \text { esterna }\end{array}$ \\
\hline Omero & $\begin{array}{l}0-2 \\
2-6 \\
6-10 \\
10-12 \\
>12\end{array}$ & $\begin{array}{l}\text { Incruenta } \\
\text { Incruenta o chirurgica } \\
\text { Incruenta o chirurgica } \\
\text { Incruenta o chirurgica } \\
\text { Incruenta o chirurgica }\end{array}$ & $\begin{array}{l}\text { Apparecchio gessato } \pm \text { trazione } \\
\text { Apparecchio gessato } \pm \text { trazione } \\
\text { Sintesi + apparecchio gessato } \pm \text { trazione } \\
\text { Fissatore esterno o sintesi } \\
\text { Sintesi interna }\end{array}$ \\
\hline Avambraccio & $\begin{array}{l}0-2 \\
2-6 \\
6-10 \\
10-12 \\
>12\end{array}$ & $\begin{array}{l}\text { Incruenta } \\
\text { Incruenta o chirurgica } \\
\text { Incruenta o chirurgica } \\
\text { Incruenta o chirurgica } \\
\text { Incruenta o chirurgica }\end{array}$ & $\begin{array}{l}\text { Apparecchio gessato } \pm \text { trazione } \\
\text { Apparecchio gessato } \pm \text { trazione } \\
\text { Apparecchio gessato } \pm \text { trazione } \\
\text { Sintesi }+ \text { apparecchio gessato } \\
\text { Sintesi + apparecchio gessato } \\
\text { o fissazione esterna }\end{array}$ \\
\hline
\end{tabular}

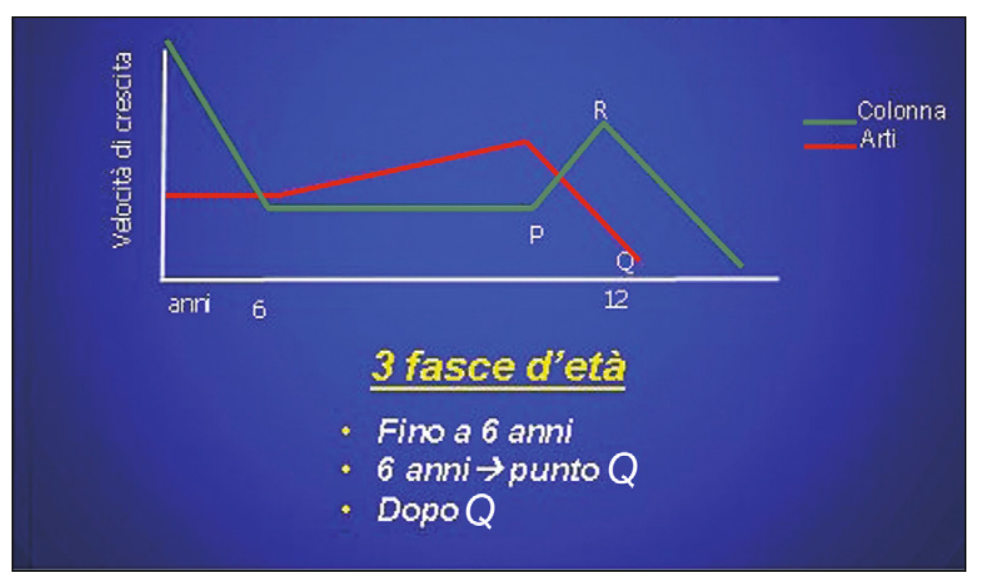

Fig. 1. Andamento con l'età della crescita di colonna e arti. Alla fine della crescita degli arti (punto 0 ) sotto l'influenza ormonale, le due curve di crescita si separano non solo affidabile, rapidamente risolutivo, Le considerazioni fin qui esposte non vogliono certo auspicare un ritorno al passato o frenare gli ulteriori futuri sviluppi tecnologici, ma semplicemente ricordare che, anche se sono mutati gli scenari migliorate le condizioni assistenziali, nulla è cambiato nella biologia dell'osso in accrescimento. Questa, in definitiva, deve rape riferimento.

In occasione del XIV Congresso Nazionale della Società Italiana di Ortopedia e Traumatologia Pebiamo cercato di fare il punto sul problema fornendo indicazioni di massima [7, 8] (Tab. 1).

Concordo pienamente nel ritenere "obbligatorio" il trattamento conservativo fino a 6 anni. Successivamente, seguendo lo schema della velocità di crescita degli arti, individuerei una fascia inter-

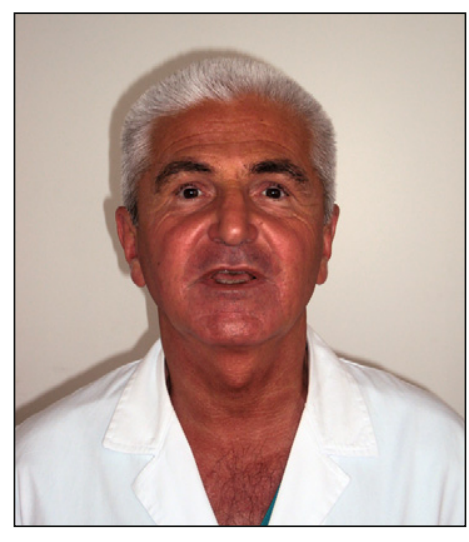

R. Facchini

media in cui ogni caso vada valutato singolarmente, specie in riferimento alle caratteristiche di peso, altezza, sviluppo delle masse muscolari, caratteri sessuali secondari, oltre che al segmento interessato, alla sede del focolaio e alla "personalità" della frattura, fino al punto $\mathrm{Q}$ (corrispondente alla fine della crescita degli arti), al di là del quale è bene optare per il trattamento chirurgico, indipendentemente dall'età anagrafica del paziente [9] (Fig. 1)

\section{Bibliografia}

Helenius I, Lamberg ST, Kääriäinen S et al (2009) Operative treatment of fractures in children is increasing. A population-based study from Finland. J Bone Joint Surg Am 91:26122616

Catalano PA, Bonizzoni C, Denti M, Facchini R (1984) Incidenza percentuale dei traumi osteoarticolari in età pediatrica presso gli Istituti Clinici di Perfezionamento di Milano. Minerva Ortopedica e Traumatologica 35:815-820

Facchini R, Denti M (1985) Disturbi dell'accrescimento degli arti inferiori nelle fratture diafisarie del bambino. Ortopedia Traumatologia Oggi 5:142-144 Denti M, Catalano PA, Bonizzoni C, Facchini R (1984) Variazioni dei tempi di consolidazione nelle fratture diafisarie del bambino in rapporto all'età. Minerva Ortopedica e Traumatologica 35:1-5

Facchini R, Albisetti W, Morandi A et al (1992) Studio statistico sullincidenza delle deformità post traumatiche degli arti inferiori. Rivista Italiana di Ortopedia e Traumatologia Pediatrica 8:400-405

Facchini R (1988). Il trattamento delle fratture diafisarie nel bambino. Minerva Ortopedica e Traumatologica 39:973976

Boero S, Catena N (2009) Trattamento incruento delle fratture diafisarie delle ossa lunghe dell'arto inferiore nei bambini fino a 6 anni. XIV Congresso Nazionale SITOP, Milano, 8-10 ottobre

Peretti G, Peretti GM (2009) Fratture diafisarie delle ossa lunghe: il trattamento in funzione delle fasce di età. XIV Congresso Nazionale SITOP, Milano, 810 ottobre

Facchini R, Morandi A, Orlando P et al (1989) Limiti di tolleranza delle scomposizioni nelle fratture diafisarie degli arti 